\title{
Avaliação da dexmedetomidina e do tramadol, associados ao midazolam, em gatas anestesiadas com isoflurano e submetidas à ovário-histerectomia
}

\author{
[Evaluation of dexmedetomidine and tramadol, associated with midazolam, in cats anesthetized \\ with isoflurane, undergoing ovariohysterectomy]
}

\author{
V.C.C. Lemos ${ }^{1}$, J.Q. Sande ${ }^{2}$, V.F. Barbosa ${ }^{3}$, J.M. Costa Neto ${ }^{3}$, E.F. Martins Filho ${ }^{3}$, C.H.D. Iwassa ${ }^{4}$ \\ ${ }^{1}$ Aluna de residência - HOSPMEV - Universidade Federal da Bahia - UFBA - Salvador, BA \\ ${ }^{2}$ Aluna de graduação - Escola de Medicina Veterinária e Zootecnia - EMEVZ - UFBA - Salvador, BA \\ ${ }^{3}$ EMEVZ - Universidade Federal da Bahia - UFBA - Salvador, BA \\ ${ }^{4}$ HOSPMEV - Universidade Federal da Bahia - UFBA - Salvador, BA
}

\begin{abstract}
RESUMO
Objetivou-se comparar as alterações cardiorrespiratórias e a analgesia pós-operatória promovidas pela dexmedetomidina e pelo tramadol, quando associados ao midazolam, em felinas. Para tal, foram selecionadas 18 gatas hígidas, divididas em dois grupos randomizados: GDM, tratadas com dexmedetomidina $(10 \mu \mathrm{g} / \mathrm{kg})$ e GTM, tratadas com tramadol $(2 \mathrm{mg} / \mathrm{kg})$, ambos associados a midazolam $(0,2 \mathrm{mg} / \mathrm{kg}$, $)$ IM. Após 15 minutos, procedeu-se à indução anestésica com propofol $(1,46 \pm 0,79 \mathrm{~mL})$, mantendo-se a anestesia com isoflurano. As felinas foram submetidas à ovário-histerectomia, registrandose as variáveis cardiorrespiratórias 15 minutos após a MPA (M0), 15 minutos após a indução (M15) e sequencialmente a cada cinco minutos, até o término do procedimento cirúrgico (M20, M25, M30, M35 e M40). A avaliação da dor iniciou-se 30 minutos após o término do procedimento cirúrgico (MP30) e sequencialmente em intervalos de 30 minutos (MP60, MP90, MP120). A partir do MP120, as avaliações foram registradas a cada hora (MP180, MP240 e MP360). A associação dexmedetomidina-midazolam infere diminuição inicial de frequência cardíaca $(\mathrm{FC})$ sem significado clínico e está relacionada à sedação mais pronunciada, à analgesia menor e menos duradoura e a episódios de êmese, quando comparada à associação tramadol-midazolam. Ambos os protocolos denotaram estabilidade cardiorrespiratória e podem ser considerados seguros em felinas submetidas à ovário-histectomia.
\end{abstract}

Palavras-chave: felina, alfa-2 agonistas, analgesia, cardiorrespiratório, opioide

\begin{abstract}
The aim of this study was to compare cardiorespiratory changes and post-operative analgesia provided by dexmedetomidine or tramadol, associated with midazolam, in female cats. For that purpose, 18 healthy cats were assigned to two randomized groups: GDM, which received dexmedetomidine (10 $\mu \mathrm{g} / \mathrm{kg}$ ) and GTM, which received tramadol (2 $\mathrm{mg} / \mathrm{kg})$, both associated with midazolam $(0.2 \mathrm{mg} / \mathrm{kg}) \mathrm{IM}$. After 15 minutes, anesthesia was induced with propofol $(1.46 \pm 0.79 \mathrm{~mL})$ and maintained with isofluorane. Ovariohysterectomy was performed and cardiorespiratory variables were registered 15 minutes after pre-anesthetic medication (M0), 15 minutes after anesthetic induction (M15), and every five minutes until the end of the surgical procedure (M20, M25, M30, M35 e M40). Pain evaluation started 30 minutes after the surgery (MP30) and sequentially at thirty-minute intervals (MP60, MP90, MP120). After MP120, each evaluation was registered at every hour (MP180, MP240 e MP360). Dexmedetomidine-midazolam association results in decreases on initial heart rate (HR) without clinical relevance and it is related to pronounced sedation, poor and less durable antinociception and vomiting events, when compared to tramadol-midazolam association. Both protocols indicate cardiorespiratoy stability and safety in cats undergoing ovariohysterectomy.
\end{abstract}

Keywords: feline, alpha 2-adrenergic agonists, analgesia, cardiorespiratory, opioid

Recebido em 15 de setembro de 2016

Aceito em 19 de janeiro de 2017

E-mail: vanessa.carolemos@hotmail.com 


\section{INTRODUÇÃO}

Considera-se a ovário-histerectomia importante medida de controle populacional realizada em felinos (Mastrocinque et al., 2006). Por constituírem prática rotineira e por representarem modelo operatório associado a dor moderada, inúmeros protocolos anestésicos têm sido testados, destacando-se especial atenção aos analgésicos, a fim de proporcionar conforto e estabilidade perioperatória.

O tramadol é um opioide atípico de ação central, que tem sido utilizado para o manejo da dor transoperatória em felinos. Apresenta-se como análogo sintético da codeína, cujos mecanismos de ação envolvem baixo grau de ligação ao receptor opioide $\mu$ e inibição central de impulsos nociceptivos nas vias descendentes espinhais, agindo na recaptação neuronal de serotonina e noradrenalina e determinando, portanto, boa eficácia analgésica (Scott e Perry, 2000). Sua administração pré-operatória $(2 \mathrm{mg} / \mathrm{kg})$ demonstrou analgesia adequada, extensiva ao pós-cirúrgico, sem ocorrência de efeitos cardiopulmonares deletérios durante esterilização cirúrgica em felinos (Cagnardi et al., 2011).

A dexmedetomidina é um representante dos alfa-2 agonistas, com ação sedativa, relaxante muscular e analgésica (Slingsby e Taylor, 2007). Seus efeitos sistêmicos incluem alterações cardiovasculares, como bradicardia, aumento da resistência vascular periférica e da pressão venosa central e redução do débito cardíaco (Monteiro et al., 2009). Apresenta-se, em doses baixas, como bom adjuvante à anestesia inalatória, ao reduzir a concentração alveolar mínima (CAM) e, consequentemente, a incidência de alterações cardiopulmonares associada aos agentes inalatórios (Pascoe, 2014).

Usualmente utilizado na medicina veterinária por seu efeito relaxante muscular, ansiolítico e hipnótico, o midazolam é frequentemente incorporado como adjuvante pré-operatório, visando ao acréscimo ao relaxamento mediado centralmente, além da ação potencializadora em protocolos multimodais, com mínimos efeitos cardiorrespiratórios (Robinson e Borer-Weir, 2015).

Em virtude das particularidades metabólicas e temperamentais do paciente felino, a abordagem anestésica multimodal mostra-se vantajosa ao associar fármacos com mecanismos diferentes, buscando-se efeitos sinérgicos associados a menores doses e consequente diminuição de efeitos colaterais (Robertson, 2008; Bellini et al., 2017).

Dessa forma, objetivou-se avaliar os efeitos cardiorrespiratórios e a analgesia pós-operatória proporcionados pelas associações dexmedetomidina-midazolam e tramadolmidazolam, em gatas anestesiadas com isoflurano, a fim de fornecer referencial para condutas analgésicas e anestésicas na espécie felina, utilizando-se a ovário-histerectomia como modelo álgico-cirúrgico.

\section{MATERIAL E MÉTODOS}

Este projeto foi aprovado pelo Comitê de Ética da Escola de Medicina Veterinária e Zootecnia da Universidade Federal da Bahia (EMEVZUFBA), sob o protocolo de número 10/2014.

Foram selecionadas 18 gatas hígidas, sem raça definida, entre cinco meses e dois anos de idade e peso médio de $2,7 \pm 0,6 \mathrm{~kg}$, classificadas como ASA I mediante avaliação clínica e laboratorial, descartando-se do estudo felinas prenhes ou em estro. Os animais foram recebidos 24 horas anteriores à realização do protocolo experimental, visando-se à adaptação ambiental, sendo mantidos em gaiolas individuais com água e alimentação ad libitum. Instituiu-se jejum alimentar de 12 horas e hídrico de duas horas prévias à abordagem anestésica.

De acordo com a medicação pré-anestésica (MPA), foram estabelecidos dois grupos randomizados, de nove animais cada, denominados: grupo GDM, tratado com $10 \mu \mathrm{g} / \mathrm{kg}$ IM de dexmedetomidina (Precedex $\AA$, Hospira) associada a $0,2 \mathrm{mg} / \mathrm{kg}$ IM de midazolam (Dormire $\AA$, Cristália), e grupo GTM, tratado com $2 \mathrm{mg} / \mathrm{kg}$ de tramadol (Tramadon $\AA$, Cristália) associado ao midazolam $(0,2 \mathrm{mg} / \mathrm{kg})$, pela mesma via. Após, procedeu-se à cateterização da veia cefálica, instituindo-se fluidoterapia com solução de ringer lactato em taxa $10 \mathrm{~mL} / \mathrm{kg} / \mathrm{h}$. Ato contínuo, as felinas receberam $30 \mathrm{mg} / \mathrm{kg}$ IV de cefalotina sódica (Cefalotina sódica, Genérico, ABL) e $0,2 \mathrm{mg} / \mathrm{kg}$ IV de meloxicam (Maxicam ${ }^{\circledR}$ $0,2 \%$, Ourofino), realizando-se sequencialmente a tricotomia da região abdominal. Decorridos 15 
minutos da MPA, procedeu-se à indução anestésica com propofol (Propovan ${ }^{\circledR}$, $1,46 \pm 0,79 \mathrm{~mL})$ e à intubação orotraqueal com sonda de Magill adequada ao porte do animal. Para a manutenção, utilizou-se isoflurano diluído em oxigênio a $100 \%$, em circuito sem reinalação de gases, em dose suficiente para suprimir resposta ao estímulo cirúrgico. Após estabilização anestésica, as gatas foram submetidas à ovário-histerectomia por incisão na linha média ventral.

Foram monitorados e coletados os dados de frequência cardíaca $(\mathrm{FC})$, frequência respiratória $(f)$, saturação de oxigênio da oxi-hemoglobina $\left(\mathrm{SpO}_{2}\right)$ e temperatura corporal $\left(\mathrm{T}^{\circ} \mathrm{C}\right)$ por leitura direta em monitor multiparamétrico (BM5 Patient Monitor, Bionet $(\mathbb{R})$. As pressões arteriais sistólica (PAS), diastólica (PAD) e média (PAM) foram obtidas em $\mathrm{mmHg}$ por meio de leitura em monitor de pressão (Deltamap ${ }^{\circledR}$, Deltalife), cujo manguito de tamanho adequado foi posicionado no membro anterior esquerdo. A pressão arterial média (PAM) foi obtida indiretamente, por meio de cálculo matemático, obedecendo-se a equação $\mathrm{PAM}=\mathrm{PAD}+[(\mathrm{PAS}-\mathrm{PAD}) / 3]$, em que PAM $=$ pressão arterial média , PAD = pressão arterial diastólica e PAS = pressão arterial sistólica. Tais variáveis foram aferidas 15 minutos após a MPA e imediatamente antes da indução anestésica (M0), 15 minutos após a indução (M15) e sequencialmente a cada cinco minutos, até o término do procedimento cirúrgico, estipulado em 25 minutos (M20, M25, M30, M35 e M40).

Após extubação, avaliação analgésica pósoperatória foi realizada por observadores treinados, que utilizaram a escala multidimensional para avaliação da dor aguda pós-operatória em gatos (Brondani et al., 2012).

A observação e a aferição das variáveis pertinentes à escala tiveram início 30 minutos após o término do procedimento cirúrgico (MP30) e novas avaliações foram realizadas em intervalos de 30 minutos (MP60, MP90, MP120). A partir do MP120, as avaliações foram registradas a cada hora (MP180, MP240 e MP360). Adicionalmente, foi notificada a ocorrência de êmese, excitação, edema pulmonar, bem como a qualidade da recuperação anestésica.
Os parâmetros avaliados na escala foram pontuados, determinando-se, sequencialmente, um escore final. Nos casos em que a somatória de escore foi igual ou superior a oito (Brondani et al., 2012), administrou-se analgesia complementar com tramadol (3mg/kg IV). A avaliação álgica pós-operatória foi realizada sempre pela mesma equipe, que se manteve cega para o protocolo utilizado na MPA.

Para a avaliação estatística das variáveis cardiorrespiratórias entre momentos de um mesmo grupo, foi utilizada a análise de variância de uma via para medidas repetidas (One way ANOVA repeated measures), e para a análise entre grupos, utilizou-se o teste $\mathrm{t}$ de Student para amostras independentes. Para a avaliação analgésica, foi utilizado o teste de Friedman para a análise de variância não paramétrica dentro de um mesmo grupo, e o teste Mann-Whitney, análogo ao teste $t$, para amostras independentes, comparando-se a média de escore entre grupos. O nível de significância adotado foi de $5 \%$.

\section{RESULTADOS E DISCUSSÃO}

No grupo tratado com dexmedetomidina (GDM), 15 minutos após a pré-medicação, 44\% dos animais apresentaram episódio de êmese, corroborando achados de outros estudos (Mcsweeney et al., 2012; Sousa et al., 2010). De forma exemplar, Slingsby et al. (2009) relataram vômito em nove dos 12 animais amostrados em seu estudo, os quais receberam o fármaco na dose $40 \mu \mathrm{g} / \mathrm{kg}$. Alterações gastrointestinais têm sido relacionadas aos alfa- 2 agonistas, tais como a êmese e a salivação, com maior incidência relacionada à xilazina, em especial nos felinos (Lemke, 2004).

Por outro lado, não foram registrados episódios de náusea ou vômito no GTM. Apesar de se tratar de reação comum durante o uso de alguns agonistas opioides $\mu$ puros, a êmese não representa um efeito típico do tramadol, provavelmente porque este atua nos receptores opioides $\mu$ e k com baixa afinidade, exercendo um fraco efeito agonista, além de bloquear a recaptação de norepinefrina e serotonina. Tais características tornam o tramadol mais vantajoso do que outros agentes opioides típicos, pelo seu perfil farmacológico único associado a uma menor incidência de efeitos adversos (Vazzana et al., 2015). 
Relativamente à avaliação cardiorrespiratória (Tab. 1), a frequência cardíaca apresentou diminuição no momento inicial, nos animais do GDM em relação ao GTM.

Os alfa-2 agonistas estão comumente associados à diminuição da atividade simpática, de modo que a bradicardia é um achado frequente, mesmo com o uso de fármacos mais modernos e seletivos, como a dexmedetomidina (Murrell e Hellebrekers, 2005). A diminuição da variável 15 minutos após a aplicação do agente sedativo está de acordo com Pypendop et al. (2011), que reportam, como principais achados hemodinâmicos relativos ao fármaco, a redução da frequência e do índice cardíaco, além de aumento da resistência vascular sistêmica. Todavia, pode-se afirmar que a associação do alfa-2 agonista com o benzodiazepínico não denotou traçados arrítmicos, excluindo-se bloqueios atrioventriculares tipicamente associados ao uso isolado do primeiro, corroborando os achados de Otero et al. (2016), que igualmente referem estabilidade do ritmo cardíaco com a associação.

Tabela 1. Valores médios $(\overline{\mathbf{X}})$ e desvio-padrão $( \pm \sigma)$ de FC (bpm), PAS, PAM e PAD $(\mathrm{mmHg}), f(\mathrm{mpm})$, $\mathrm{SpO}_{2}(\%)$ e $\mathrm{T}\left({ }^{\circ} \mathrm{C}\right)$ em gatas pré-tratadas com dexmedetomidina-midazolam (GDM) ou tramadolmidazolam (GTM) e submetidas à ovário-histerectomia

\begin{tabular}{|c|c|c|c|c|c|c|c|c|}
\hline \multirow{2}{*}{ Variável } & \multirow{2}{*}{ Grupos } & \multicolumn{7}{|c|}{ Momentos } \\
\hline & & M0 & M15 & M20 & M25 & M30 & M35 & M40 \\
\hline \multirow{2}{*}{$\begin{array}{c}\mathrm{FC} \\
(\mathrm{bpm})\end{array}$} & GDM & $115 \pm 40 \mathrm{~A}$ & $134 \pm 15$ & $142 \pm 20$ & $144 \pm 17$ & $146 \pm 18$ & $147 \pm 18$ & $144 \pm 18$ \\
\hline & GTM & $185 \pm 23 \mathrm{Ba}$ & $131 \pm 16 b$ & $152 \pm 22 b$ & $148 \pm 16 b$ & $150 \pm 7 b$ & $153 \pm 15$ & $141 \pm 14 b$ \\
\hline \multirow{2}{*}{$\begin{array}{c}\text { PAS } \\
(\mathrm{mmHg})\end{array}$} & GDM & $136 \pm 28 a$ & $111 \pm 24$ & $119 \pm 25$ & $106 \pm 23$ & $94 \pm 18 b$ & $103 \pm 19$ & $103 \pm 31$ \\
\hline & GTM & $130 \pm 26$ & $97 \pm 24$ & $115 \pm 23$ & $110 \pm 17$ & $108 \pm 19$ & $101 \pm 28$ & $123 \pm 31$ \\
\hline \multirow{2}{*}{$\begin{array}{c}\text { PAM } \\
(\mathrm{mmHg})\end{array}$} & GDM & $110 \pm 30 \mathrm{a}$ & $75 \pm 20$ & $82 \pm 20$ & $82 \pm 22$ & $72 \pm 16 b$ & $75 \pm 20$ & $81 \pm 25$ \\
\hline & GTM & $94 \pm 21$ & $67 \pm 12$ & $93 \pm 22$ & $77 \pm 14$ & $81 \pm 17$ & $77 \pm 22$ & $85 \pm 26$ \\
\hline \multirow{2}{*}{$\begin{array}{c}\text { PAD } \\
(\mathrm{mmHg})\end{array}$} & GDM & $97 \pm 33 a$ & $57 \pm 23$ & $64 \pm 24$ & $70 \pm 23$ & $61 \pm 18 b$ & $65 \pm 16$ & $70 \pm 28$ \\
\hline & GTM & $86 \pm 17 a$ & $51 \pm 11 b$ & $82 \pm 23$ & $61 \pm 18$ & $68 \pm 18$ & $65 \pm 21$ & $62 \pm 30$ \\
\hline \multirow{2}{*}{$\begin{array}{c}f \\
(\mathrm{mpm})\end{array}$} & GDM & $48 \pm 13 a$ & $27 \pm 9 b$ & $23 \pm 5 b$ & $23 \pm 5 b$ & $25 \pm 4 b$ & $26 \pm 6 b$ & $31 \pm 22$ \\
\hline & GTM & $68 \pm 35 a$ & $27 \pm 10 b$ & $27 \pm 8 b$ & $26 \pm 6 b$ & $24 \pm 5 b$ & $23 \pm 6 b$ & $26 \pm 7 b$ \\
\hline \multirow{2}{*}{$\begin{array}{c}\mathrm{SpO}_{2} \\
(\%)\end{array}$} & GDM & $98,2 \pm 0,8$ & $97,8 \pm 2,1$ & $98,1 \pm 1,1$ & $97,3 \pm 2,1 \mathrm{~A}$ & $98,2 \pm 1,6$ & $97,6 \pm 1,7$ & $98,4 \pm 0,7$ \\
\hline & GTM & $97 \pm 2,8$ & $98,8 \pm 0,3$ & $98,4 \pm 0,7$ & $99,0 \pm 0 \mathrm{~B}$ & $98,7 \pm 0,4$ & $98,6 \pm 0,9$ & $98,6 \pm 0,6$ \\
\hline \multirow{2}{*}{$\begin{array}{c}\mathrm{T} \\
\left({ }^{\circ} \mathrm{C}\right)\end{array}$} & GDM & $38,5 \pm 0,7 \mathrm{a}$ & $37,8 \pm 1,0 \mathrm{~b}$ & $37,3 \pm 1,1 b$ & $37,0 \pm 1,1 \mathrm{~b}$ & $36,8 \pm 1,1 b$ & $36,7 \pm 1,1 b$ & $36,9 \pm 0,8 b$ \\
\hline & GTM & $38,4 \pm 0,5 \mathrm{a}$ & $37,6 \pm 0,8 b$ & $37,6 \pm 0 \mathrm{~b}$ & $37,2 \pm 0,3 b$ & $37,0 \pm 0,3 b$ & $36,9 \pm 0,3 b$ & $37,0 \pm 0,3 b$ \\
\hline
\end{tabular}

Médias seguidas por letras minúsculas diferentes nas linhas demonstram diferença estatística entre momentos $(\mathrm{P}<0,05)$. Médias seguidas por letras maiúsculas diferentes nas colunas demonstram diferença estatística entre grupos $(\mathrm{P}<0,05)$.

Adicionalmente, foi observada redução significativa da FC no GTM, 15 minutos após a indução com propofol, fato possivelmente associado à diminuição da atividade simpática pelo anestésico geral intravenoso, conforme relato de outros autores (Amengual et al., 2013). Tais achados corroboram os de Bellini et al. (2017), que reportaram similar diminuição da variável após o uso do propofol, em gatas tratadas com tramadol, buprenorfina ou ambos. Contudo, apesar da variação, não foi extrapolado o limite de normalidade para a espécie, considerado entre 100 - 200bpm (Muir III, 2012).

Outros estudos demonstram alterações cardíacas mínimas com o uso do tramadol (Borges et al., 2008; Cagnardi et al., 2011) e, na presente pesquisa, acredita-se que o midazolam tenha contribuído para tal estabilidade, visto que tipicamente não está vinculado a alterações cardiorrespiratórias significativas (Robinson e Borer-Weir, 2015). 
Relativamente à avaliação transoperatória das pressões arteriais, não foram observadas diferenças entre os tratamentos (Tab. 1). No grupo que recebeu o alfa-2 agonista, houve redução significativa das variáveis em M30 relativamente ao $\mathrm{M} 0$.

A dexmedetomidina apresenta reconhecido efeito bifásico sobre a pressão arterial, inicialmente vinculado à vasoconstrição transitória associada ao declínio de FC, para posterior estabelecimento de hipotensão duradoura (Murrell e Hellebrekers, 2005). A associação com midazolam não impediu as oscilações pressóricas, contudo podese afirmar que as médias foram mantidas próximas ao intervalo considerado fisiológico (Muir III, 2012). Em cães, a associação da dexmedetomidina com o midazolam, em infusão contínua, resultou em maior estabilidade hemodinâmica em relação ao alfa-2 agonista infundido isoladamente (Otero et al., 2016).

O GTM denotou maior estabilidade pressórica, evidenciando apenas diminuição da PAD 15 minutos após a indução anestésica, fato que vem ao encontro do já referenciado efeito do propofol sobre o sistema nervoso simpático. Contudo, a variação não se reveste de importância clínica. A estabilidade dos valores de pressão arterial com o uso do tramadol tem sido relatada em gatos, em que se utilizou a mesma dose que o presente trabalho (Teppema et al., 2003).

Os diferentes tratamentos não imprimiram diferença na $f$ (Tab. 1), embora o GDM tenha apresentado menores médias no momento inicial, provavelmente em função do maior efeito sedativo imputado à associação do midazolam com o alfa-2 agonista, quando comparado à associação com o opioide.

$\mathrm{Na}$ análise entre os momentos, observou-se, em ambos os grupos, redução dos valores após a indução anestésica, devido à reconhecida ação depressora do propofol sobre centro respiratório (Blouin et al., 1993). Contudo, as médias permaneceram estáveis e dentro do normal, descartando-se eventos hipoxêmicos, conforme atestam as médias de oximetria.

A dexmedetomidina não está associada à depressão respiratória em felinos, mesmo considerando-se doses maiores $(25-75 \mu \mathrm{g} / \mathrm{kg})$ que a do trabalho em pauta (Ansah et al., 1998). No que se refere ao tramadol, estudos em felinos demonstraram efeito depressor dose-dependente e passível de reversão pela naloxona (Teppema et al., 2003). Todavia, a dose do opioide utilizada é considerada segura por outros autores, corroborando os presentes achados (Cagnardi et al., 2011; Bellini et al., 2017). Por fim, para ambos os protocolos, não houve interferência depressora do midazolam, conforme se espera com uso de benzodiazepínicos que classicamente não interferem nas variáveis respiratórias (Covey-Crump e Murison, 2008).

A $\mathrm{T}^{\circ} \mathrm{C}$ não diferiu entre os grupos estudados, havendo apenas diminuição ao longo do tempo. Apesar do uso do colchão térmico ativo, a mínima perda de calor constatada ocorreu provavelmente em razão dos efeitos da anestesia geral, que incluem miorrelaxamento, vasodilatação e depressão do centro termorregulador (Clarke et al., 2014). A diminuição da temperatura em gatas foi reportada em estudo em que o tramadol foi comparado à petidina, porém os autores igualmente imputaram a perda de calor aos efeitos do anestésico eleito (isoflurano) sobre a termorregulação (Evangelista et al., 2014).

A observação clínica pós-operatória das felinas denotou menores efeitos adversos com o uso da combinação tramadol-midazolam, visto que os animais apresentaram recuperação mais rápida e satisfatória, embora alguns animais demonstrassem excitação, evento frequentemente associado ao uso de opioides em felinos (Steagall et al., 2008). Contudo, essa alteração comportamental não foi considerada relevante, havendo rápida remissão dos sinais excitatórios.

Em contrapartida, os animais do grupo GDM apresentaram maior ocorrência de efeitos indesejáveis, como recuperação prolongada e sedação mais pronunciada nos momentos iniciais da avaliação pós-operatória.

A análise estatística demonstrou diferença significativa entre os escores dos grupos apenas no MP120, ou seja, duas horas após o procedimento cirúrgico, apesar de serem observadas maiores médias nos animais tratados com o alfa-2 agonista (Tab. 2). 
Tabela 2. Valores médios e desvios-padrão (s) do escore de dor, de acordo com escala multidimensional, em gatas pré-tratadas com dexmedetomidina-midazolam (GDM) ou tramadol-midazolam (GTM) e submetidas à ovário-histerectomia

\begin{tabular}{cccccccc}
\multirow{2}{*}{ Grupos } & \multicolumn{7}{c}{ Momentos } \\
\cline { 2 - 7 } & MP30 & MP60 & MP90 & MP120 & MP180 & MP240 & MP360 \\
\hline GDM & $8 \pm 3^{\mathrm{a}}$ & $7 \pm 3$ & $8 \pm 4^{\mathrm{a}}$ & $10 \pm 6^{\mathrm{Ba}}$ & $8 \pm 6$ & $5 \pm 4$ & $2 \pm 3^{\mathrm{b}}$ \\
GTM & $6 \pm 4$ & $6 \pm 4^{\mathrm{a}}$ & $5 \pm 4$ & $4 \pm 4^{\mathrm{A}}$ & $4 \pm 3$ & $3 \pm 3$ & $3 \pm 3^{\mathrm{b}}$ \\
\hline
\end{tabular}

Médias seguidas por letras minúsculas diferentes nas linhas demonstram diferença estatística entre momentos $(\mathrm{P}<0,05)$. Médias seguidas por letras maiúsculas diferentes nas colunas demonstram diferença estatística entre grupos $(\mathrm{P}<0,05)$.

Os animais tratados do GDM obtiveram escores crescentes até 120 minutos após o procedimento cirúrgico, evidenciados pelas médias estatisticamente maiores dos momentos MP30, MP90 e MP120 em relação ao MP360 $(\mathrm{P}=0,0081)$. Assim, o resgate analgésico foi necessário em oito dos nove animais tratados até o M120 e em 100\% dos animais, considerandose todo o período de avaliação. Em contrapartida, o GTM evidenciou melhor recuperação anestésica, visto que não houve necessidade de intervenção analgésica em 44\% dos animais, em nenhum momento da avaliação pós-operatória (Tab. 3). Ademais, a necessidade de resgate analgésico (escore $\geq 8$ ) apresentada pelo grupo GDM foi, em média, aos 87 minutos após o término da cirurgia, o que demonstra notável contraste com a média do GTM de 180 minutos. Acredita-se, dessa forma, que o efeito analgésico da dexmedetomidina não se estenda ou mostre-se insuficiente além do período supracitado, destacando-se a melhor cobertura analgésica dos animais tratados com o tramadol associado ao midazolam, durante toda a avaliação pósoperatória.

Tabela 3. Necessidade de analgesia de resgate (escore de dor $\geq 8$ ), de acordo com escala multidimensional, em gatas pré-tratadas com dexmedetomidina-midazolam (GDM) ou tramadol-midazolam (GTM) e submetidas à ovário-histerectomia.

\begin{tabular}{ccc}
\hline Grupo & Escore $\geq 8$ & Escore inferior a 8 \\
\hline GDM & 9 & 0 \\
\hline GTM & 5 & 4 \\
\hline
\end{tabular}

De acordo com Mcsweeney et al. (2012), em estudo realizado com felinos submetidos a procedimentos cirúrgicos de curta e longa duração, a dexmedetomidina, utilizada na MPA, demonstrou período analgésico hábil de, no máximo, duas a quatro horas, contudo a dose utilizada foi de $40 \mu \mathrm{g} / \mathrm{kg}$ (IM), consideravelmente maior que a do estudo em tese. Outro estudo, que utilizou a mesma dose, afirma que esse fármaco, unicamente na dose $40 \mu \mathrm{g} / \mathrm{kg}$, foi capaz de fornecer cobertura analgésica suficiente, bem como relata insuficiência das doses 5 a $20 \mu \mathrm{g} / \mathrm{kg}$ em elevar o limiar nociceptivo. Contudo, o mesmo estudo indica um elevado potencial das doses mais altas em mascarar a hiperalgesia em razão da sedação pronunciada exercida pelas doses superiores a $20 \mu \mathrm{g} / \mathrm{kg}$ (Slingsby et al., 2007). Assim, os autores recomendam considerar o uso de outros analgésicos pós-operatórios de acordo com o tipo de procedimento, a severidade da dor e a dose analgésica da dexmedetomidina pré-administrada.

No que se refere ao uso do opioide associado ao midazolam, os momentos MP60 e MP360 obtiveram diferenças significativas, com maior média observada no MP60, momento no qual cinco das nove gatas demandaram resgate analgésico. Desse modo, com base nos achados, a analgesia observada no GTM, embora mais significativa que do GDM, não se apresentou tão relevante como em outros estudos que atestam a eficácia analgésica do tramadol $(2 \mathrm{mg} / \mathrm{kg}) \mathrm{em}$ gatos submetidos à gonadectomia, descartandose o uso de resgate analgésico (Cagnardi et al., 2011). Outros estudos em cadelas demonstraram que o uso preemptivo desse opioide, em dose consideravelmente maior $(6 \mathrm{mg} / \mathrm{kg} \mathrm{IM})$, reduziu a dor pós-operatória relativa à ovário-histerectomia durante as três primeiras horas após a extubação (Rodrigues et al., 2013). 


\section{CONCLUSÕES}

Com bases nos dados obtidos neste estudo, a associação do midazolam com o tramadol ou com a dexmedetomidina não promoveu alterações cardiorrespiratórias importantes; no entanto a associação dexmedetomidinamidazolam reduz a frequência cardíaca $(\mathrm{FC})$ e promove sedação mais pronunciada, episódios de êmese e analgesia mais fraca e menos duradoura, quando comparada à associação tramadolmidazolam.

\section{AGRADECIMENTOS}

À Fapesb - Fundação de Amparo à Pesquisa do Estado de Bahia - e ao CNPq - Conselho Nacional de Desenvolvimento Científico e Tecnológico -, pelo fomento em forma de bolsa de estudo, por meio do Programa Institucional de Bolsas de Iniciação científica - PIBIC; à Universidade Federal da Bahia; à Pró-Reitoria de Pesquisa, Criação e Inovação e a Pró-Reitoria de Ensino de Pós-Graduação, pelo apoio ao projeto de pesquisa, por meio do Programa de Apoio a Pesquisadores Emergentes da UFBA (Prodoc/UFBA).

\section{REFERÊNCIAS}

AMENGUAL, M.; FLAHERTY, D.; AUCKBURALLY, A. et al. An evaluation of anaesthetic induction in healthy dogs using rapid intravenous injection of propofol or alfaxalone. Vet. Anaesth. Analg., v.40, p.115-123, 2013.

ANSAH, O.B.; RAEKALLIO, M.; VAINIO, O. Comparison of three doses of dexmedetomidine with medetomidine in cats following intramuscular administration. J. Vet. Pharmacol. Therap., v.21, p.380-387, 1998.

BELLINI, L.; MOLLO, A.; CONTIERO, B.; BUSETTO, R. Intraoperative end-tidal concentration of isoflurane in cats undergoing ovariectomy that received tramadol, buprenorphine or a combination of both. $J$. Feline Med. Surg., v.19, n.2, p.110-116, 2017.

BLOUIN, R.T.; SEIFERT, H.A.; BABENCO H.D. et al. Propofol depresses the hypoxic ventilatory response during conscious sedation and isohypercapnia. Anesthesiology, v.79, p.1177-1182, 1993.
BORGES, P.; NUNES, N.; BARBOSA, V.F. et al. Variáveis cardiorrespiratórias, índice bispectral e recuperação anestésica em cães anestesiados pelo isoflurano, tratados ou não com tramadol. Arq. Bras. Med. Vet. Zootec., v.60, p.613-619, 2008.

BRONDANI, J.T.; LUNA, S.P.L; MINTO, B.W. et al. Validade e responsividade de uma escala multidimensional para avaliação de dor pósoperatória em gatos. Arq. Bras. Med. Vet. Zootec., v.64, p.1529-1538, 2012.

CAGNARDI, P.; GALLO, M.; BECCAGLIA, M. et al. Pharmacokinetics, intraoperative effect and postoperative analgesia of tramadol in cats. Res. Vet. Sci., v.90, p.503-509, 2011.

CLARKE, K.W.; HALL, L.W.; TRIM C.M. Patient monitoring and clinical measurement. In: CLARKE, K.W.; TRIM C.M. Veterinary anaesthesia. 11.ed. [Amesterdã], Elsevier, 2014. p.19-63.

COVEY-CRUMP, G.L.; MURISON P.J. Fentanyl or midazolam for co-induction of anaesthesia with propofol in dogs. Vet. Anaesth. Analg., v.35, p.463-472, 2008.

EVANGELISTA, M.C.; SILVA, R.A.; CARDOZO, L.B. et al. Comparison of preoperative tramadol and pethidine on postoperative pain in cats undergoing ovariohysterectomy. BMC Vet. Res., n.10, p.252260, 2014.

LEMKE, K.A. Perioperative use of selective alpha-2 agonists and antagonists in small animals. Can. Vet. J., v.45, p.475-480, 2004.

MASTROCINQUE, S.; IMAGAWA V.H.; ALMEIDA T.F. et al. Gonadectomia em gatas impúberes. Técnica anestésica. Braz. J. Vet. Res. Anim. Sci., v.43, p.810-815, 2006.

MCSWEENEY, P.M.; MARTIN, D.D.; RAMSEY, D.S.; MCKUSICK, B.C. Clinical efficacy and safety of dexmedetomidine used as a preanesthetic prior to general anesthesia in cats. J. Am. Vet. Med. Assoc., v.240, p.404-412, 2012.

MONTEIRO, E.R.; CAMPAGNOL, D.; PARRILHA, L.R.; FURLAN, L. Evaluation of cardiorespiratory effects of combinations of dexmedetomidine and atropine in cats. J. Feline Med. Surg., v.11, p.783-792, 2009. 
MUIR III, W.W. Animal evaluation and preparation. In: MUIR III, W.W.; HUBBELL, J.A.E.; BEDNARSKI， R.; LERCHE， P. Handbook of veterinary anesthesia. 5.ed. St. Louis: Mosby, 2012. p.11-22.

MURRELL, J.C.; HELLEBREKERS, L.J. Medetomidine and dexmedetomidine: a review of cardiovascular effects and antinociceptive properties in the dog. Vet. Anaesth. Analg., v.32, p.117-127, 2005.

OTERO, A.R.S.; BARBOSA, V.F.; CARNEIRO, R.L. et al. Avaliação da infusão contínua de dexmedetomidina ou dexemedetomidina-midazolam sobre variáveis cardiorrespiratórias e qualidade da recuperação anestésica, em cadelas submetidas à ovariosalpingohisterectomia. Rev. Bras. Med. Vet., v.38, p.168-174, 2016.

PASCOE, P.J. The cardiopulmonary effects of dexmedetomidine infusions in dogs during isoflurane anesthesia. Vet. Anaesth. Analg., p.19, 2014.

PYPENDOP, B.H.; BARTER, L.; SCOTT, D.; ILKIW, J. Hemodynamic effect of dexmedetomidine in isoflurane-anesthetized cats. Vet. Anaesth. Analg., v.38, p.555-567, 2011.

ROBERTSON, S.A. Managing Pain in Feline Patients. Vet. Clin. N. Am. Small Anim. Prac., v.38, p.1267-1290, 2008.

ROBINSON, R.; BORER-WEIR, K. The effects of diazepam or midazolam on the dose of propofol required to induce anaesthesia in cats. Vet. Anaesth. Analg., p.1-9, 2015.

RODRIGUES, C.M.; LIMA, P.; CRUZ, T. et al. Analgesia preemptiva com cloridrato de tramadol em ovariohisterectomia de cadelas. In: SEMINÁRIO DE INICIAÇÃO CIENTÍFICA, 21., Ijuí. Anais... Ijuí: Unijuí, 2013. p.4-7, 2013.
SCOTT, L.J.; PERRY, C.M. Tramadol: a review of its use in perioperative pain. Drugs, v.60, p.139-176, 2000.

SLINGSBY, L.S.; TAYLOR, P.M. Thermal antinociception after dexmedetomidine administration in cats: a dose-finding study. $J$. Vet. Pharmacol. Therap., v.31, p.135-142, 2007.

SLINGSBY, L.S.; TAYLOR, P.M.; MONROE, T. Thermal antinociception after dexmedetomidine administration in cats: a comparison between intramuscular and oral transmucosal administration. J. Feline Med. Surg., v.11, p.829-834, 2009.

SOUSA, S.S.; INTELISANO, T.; DE BIAGGI, C. et al. Cardiopulmonary and isoflurane-sparing effects of epidural or intravenous infusion of dexmedetomidine in cats undergoing surgery with epidural lidocaine. Vet. Anaesth. Analg., v.37, p.106-115, 2010.

STEAGALL, P.; TAYLOR, P.; BRONDANI, J. et al. Antinociceptive effects of tramadol and acepromazine in cats. J. Feline Med. Surg., v.10, p.24-31, 2008.

TEPPEMA, L.J.; NIEUWENHUIJS, D.; OLIEVIER, C.; DAHAN, A. Respiratory depression by tramadol in the cat. Anesthesiology, v.98, p.420-427, 2003.

VAZZANA, M.; ANDREANI, T.; FANGUEIRO, J. et al. Tramadol hydrochloride: pharmacokinetics, pharmacodynamics, adverse side effects, co-administration of drugs and new drug delivery systems. Biomed. Pharmacother., v.70, p.234-238, 2015. 